potentially impacting outcomes. We retrospectively analysed a cohort of patients who survived an $\mathrm{AD}$ event and were offered specialist clinic follow up in our centre.

Methods Electronic records were reviewed for all patients surviving an admission for $\mathrm{AD}$ and offered rapid local follow up (within 4 weeks), from January 2016 to December 2017. Demographic data, clinic attendances and clinical course (Child-Pugh/MELD scores at 1, 3, 6, and 12 months) were recorded, as well as 1-year mortality and abstinence rates.

Results 129 unique patients were included (32\% female; 68\% male), with median age 53 (range 29-83). Mortality was 24\% $(\mathrm{n}=31)$ at 1 year, in keeping with published cohorts. Of these, $55 \%(\mathrm{n}=17)$ were directly attributable to liver disease. At 3 years mortality reached $51 \%(\mathrm{n}=66)$, with approximately half $(48 \% ; n=32)$ due to a liver cause. $91 \mathrm{AD}$ survivors $(71 \%)$ attended their initial appointment (non-attendance rate 29\%). 1 year mortality was significantly lower in this group compared to non-attenders [18 (20\%) v $14(38 \%), \mathrm{p}=0.03]$. This was reflected in a downward trend in mean MELD scores for attenders at 1, 3, 6 and 12 months $(15,15,14$ and 12 respectively), whereas non-attenders showed static or worsening disease $(14,15,17,14)$. Similarly, Child-Pugh (CP) scores showed significantly greater numbers of attenders recompensated to CP-A by 12 months [29 (32\%) v 5 (16\%), p=0.033]. Nearly half $(47 \% ; n=60)$ of all patients were still drinking alcohol at 1 year. However, those who attended the first appointment were significantly more likely to be abstinent at 1 year than those who did not $[42(46 \%)$ v $6 \quad(16 \%)$, $\mathrm{p}=0.002]$, which is considered the most likely reason for better 1-year survival in this group.

Conclusions Our results show that poor initial engagement with follow up is very common, and is associated with lower rates of abstinence, recompensation, and survival at 1 year. There are likely to be many reasons patients cannot or do not attend. Exploring these factors - and identifying ways to promote engagement - should be considered a high priority to improve longer-term outcomes.

\section{PWE-5 EVALUATION OF INFECTION STATUS IN PATIENTS PREVIOUSLY DIAGNOSED WITH HEPATITIS C IN A LOCAL AUTHORITY}

${ }^{1}$ Michael Zhang*, ${ }^{2}$ Muhammad Ammar bin Abd Wahab, ${ }^{1}$ Athar Saeed. 'Gateshead Health NHS Foundation Trust, Gateshead, UK; ${ }^{2}$ Newcastle University Medicine Malaysia, Gelang Patah, Malaysia

\subsection{6/gutjnl-2021-BSG.195}

Introduction The World Health Organization aims to eliminate the threat of hepatitis C (HCV) by 2030. Fortunately, there are now highly effective direct acting antiviral (DAA) drugs that can be used to treat the virus. Despite this, patients with untreated or incomplete treatment of HCV persist. We aim to identify patients within a local authority who have active HCV infection in order re-engage them into our services.

Methods A retrospective review of patients diagnosed with hepatitis C between 1996 and 2017 was conducted in a single local authority. The patients were identified from a Public Health England database. The data was originally collected in July 2019 and reviewed in November 2020. Those identified as having active HCV infection were called to arrange confirmatory RNA testing with a view to starting treatment if found positive. All other patients were reviewed for changes to their
Abstract PWE-5 Table 1 Number of HCV patients classified based on their infection status in July 2019 and November 2020

\begin{tabular}{lll}
\hline & Jul 2019 & Nov \\
& & 2020 \\
\hline A & 30 & 27 \\
& $(25.8 \%)$ & $(24.3 \%)$ \\
B1 & $7(6.0 \%)$ & $4(3.6 \%)$ \\
B2 & $10(8.6 \%)$ & 12 \\
& & $(10.8 \%)$ \\
B3 & $9(7.8 \%)$ & 12 \\
& & $(10.8 \%)$ \\
C & 60 & 56 \\
& $(51.7 \%)$ & $(50.5 \%)$ \\
Total & 116 & 111 \\
\hline
\end{tabular}

infection status. Patients who had moved out of area or were deceased were excluded from analysis.

Results A total of 111 patients were included in November 2020. The patients were classified into three groups. Group A represented patients with ongoing HCV infection and accounted for 27 (24.3\%) patients. Within Group A, 12 were being actively managed, 10 were uncontactable, 4 moved out of area and one was unwilling to engage. Group B included those who required further investigation to determine their infection status, accounting for 28 (25.2\%) patients. Amongst these: 4 patients had a positive HCV antibody test without any record of RNA PCR testing (Group B1); 12 had no record of ever having $\mathrm{HCV}$ infection (Group B2); and 12 required further RNA PCR testing to confirm HCV clearance post-treatment (Group B3). Group C patients were clear of HCV infection and accounted for 56 (50.5\%) patients. This data is compared to data from July 2019 in Table 1. There were 7 cases of re-infection and 10 patients were deceased. Conclusions The majority of patients are managed appropriately. However, the proportion of patients requiring further management has remained static over the past year. Nonattendance and difficulty contacting patients remain predominant barriers to management. Different methods such as improved co-ordination with community services, flexible appointments and opportunistic testing are needed in this local authority to achieve the WHO target of eliminating HCV by 2030. The feasibility and efficacy of this will be evaluated in 6 months' time.

\section{PWE-6 BIOLOGIC INDUCED HEPATOTOXICITY IN INFLAMMATORY BOWEL DISEASE (IBD); A SYSTEMATIC REVIEW AND META-ANALYSIS}

${ }^{1}$ Eyad Gadour*, 'Zeinab Hassan. 'University Hospitals of Morecambe Bay NHS Foundation Trust, Manchester, UK; ${ }^{2}$ The National Ribat University-Faculty of Medicine, Khartoum, Sudan

\subsection{6/gutjnl-2021-BSG.196}

Introduction Hepatotoxicity and use of biologic drugs have historically been challenging in IBD. We aim to study the prevalence of hepatotoxicity in adult patients using biologic medications.

Methodology With the guidelines described by PRISMA-P, a detailed search strategy for each electronic database were 